reign colful experiprevalent if the four mfident of The Year

\title{
The Importance of the GHO in General Practice
}

\author{
P F M VERHAAK, H J WENNINK AND M A R TIJHUIS \\ Verhaak P F M, Wennink H J and Tijhuis M A R. The importance of the GHQ in general practice. Family
Practice 1990; 7: 319-324. \\ The relationship between General Health Questionnaire (GHQ) score and complaints presented at the \\ as might be expected. Many patients \\ low GHQ score; many patients with who present psychosocial problems to their GP appear to have a \\ also assessed by the GP as being a high GHO score exclusively present somatic complaints, which are
}

\section{INTRODUCTION AND PRESENTATION OF THE QUESTION}

Most people with mental disorders [defined as the presence of symptoms and/or signs which originate from or are sustained by emotional conflicts and/or stressing conditions. (cf Ref 10)] are not treated by the health care services. ${ }^{1,2}$ This is often put down to the fact that a GP does not always identify the true nature of the complaint. As a solution, the use of screening instruments in primary care has been suggested, and the General Health Questionnaire (GHQ) has been frequently mentioned.

The GHQ is used as a first stage screening instrument for the identification of mental disorders, ${ }_{1,3-10}$ and measures the likelihood of a person being classified a psychiatric 'case'. Given a standardized clinical interview (eg the Present State Examination) as a 'gold standard', the GHQ has proved to be a specific, sensitive instrument ${ }^{6,10-14}$ and has been used successfully in clinical and general populations. ${ }^{4}$ Hodiamont and Veling ${ }^{15}$ came to the conclusion that the GHQ is an efficient questionnaire for estimating the prevalence of serious psychiatric problems in a specific population. Tarnopolsky et al. ${ }^{12}$ concluded that in the region of $15-30 \%$ high GHQ scores, where the results of most surveys are expected to fall, the GHQ predicts with reasonable closeness the proportion of psychiatric disorders'

These characteristics may be sufficient to make a reliable estimation of the probability that a patient is a psychiatric case, but it remains questionable whether screening for mental disorders in primary care is useful at all. The GHQ measures the prevalence of psychiatric complaints in the population: a GP reacts pri-

Netherlands Institute for Primary Health Care (NIVEL), PO Box 1568-3500 BN Utrecht, The Netherlands. marily to the demand for assistance at a specific moment in time. We are dealing, therefore, with the difference between epidemiological and clinical medicine, between screening and diagnosing. A patient who requests an analgesic for influenza, may also be fering the doctors as a diabetic, or as a person suffering from severe strain, but at the moment of the GHQ detects 'psychiatic which is presented. The GHQ detects 'psychiatric cases' regardless of the is primarily guided byeds. The GP on the other hand, primarily guided by those needs.

Moreover, the GHQ represents a specialist point of view, whereas the GP has a generalistic point of view. The difference between the two is sometimes charac terized as: 'treat unless' as opposed to 'do not treat unless'. This difference can clearly be seen in the complaints which psychiatrists raise about GPs failing to identify serious psychiatric problems (compare the studies cited above), but it also explains the GPs complaint that the psychiatric point of view does not fit in with their way of looking at things. ${ }^{16,17}$ This also raises the question of whether, in principle, GPs should follow up matters which they suspect, but which are not presented to them by the patient. In other words, one to transfer to wheening it is useful or desirable for a GP to transfer to screening and the active detection of psy-

To decide about the feasibility of the GHQ as an instrument for screening in general practice, we need to know how GHQ-cases present themselves in general practice. A second question is to what in patients who present with question is to what degree patients who present with mental problems in general psychologially diagnosed by the GP as having GHQ.

Such a description might contribute to an insight in the practical relevance of screening for general practice. 


\section{METHOD}

The basic material used to answer these questions consisted, on the one hand, of the GHQ-30 and, on the other hand, of data concerning the presented complaints, and the assessment of the situation by the GP. These data were collected within the framework of a Morbidity and Intervention Survey in General Practice, conducted by the NIVEL. For this study a representative sample survey of Dutch general practices $(n=160)$ was selected and all contacts with patients were registered over a period of 3 months. In addition 100 patients per practice were interviewed; this interview included filling in of GHQ-30 (for further details of this survey see Foets, Van der Velden and Van der $\left.\mathrm{Zee}^{18}\right)$. Using this data as a basis it was possible to compare the GHQ scores and the nature of the complaint as recorded in the practice notes.

Having taken the term GHQ score to be a known entity, we must now turn to the way in which the presentation of the problem and the assessment was determined in the morbidity study mentioned. This took place on the basis of the complaint as expressed by the patient and on the basis of the subsequent assessment recorded by the doctor. These variables were registered by the GP during each consultation on a so-called 'reason for encounter form'. Two complaint aspects were assessed on this form.

\section{Reason for encounter}

We are dealing here with the complaint presented in the words of the patient. For our analysis we distinguish two groups: complaints which are partly of a psychosocial nature (eg feeling depressed, fear, cannot sleep, relationship problems), and complaints whereby this is not the case (eg cough, stomach ache). The complaint was recorded verbatim by the GP and later coded with the aid of the so-called ICPC coding. ${ }^{19}$ Complaints from chapter ' $\mathrm{P}$ ' (psychological) and ' $\mathrm{Z}$ ' (social) were considered 'psychosocial' and others somatic by presentation.

\section{Assessment by the doctor}

Due to the fact that the ICPC coding system is restricted to a single axis classification in organ systems, the

TABLE 1 GHQ sum scores, in the total sample survey of the National Study and in the week's sample survey

\begin{tabular}{lcc}
\hline GHQ sum score & $\begin{array}{c}\text { National study } \\
(n=8747)\end{array}$ & $\begin{array}{c}\text { Week sample } \\
(n=476)\end{array}$ \\
\hline 0 & $60 \%$ & $48 \%$ \\
1 & $14 \%$ & $17 \%$ \\
2 & $6 \%$ & $9 \%$ \\
3 & $4 \%$ & $3 \%$ \\
4 & $3 \%$ & $4 \%$ \\
5 & $2 \%$ & $2 \%$ \\
6 & $1 \%$ & $2 \%$ \\
7 & $1 \%$ & $2 \%$ \\
8 & $1 \%$ & $1 \%$ \\
9 & $1 \%$ & $1 \%$ \\
10 & $6 \%$ & $11 \%$ \\
\hline
\end{tabular}

extent to which psychosocial problems are present (eg anxiety, underlying vague complaints; stress causing gastritis) cannot be expressed within this system. This is why each complaint was also assessed by the GP on a five-point scale ranging from entirely somatic to entirely mental. In this way complaints presented as somatic can also be assessed as contributory factors to eventual mental problems.

These two aspects make it possible to distinguish three relevant categories within the scale of problems presented to the GP: complaints which are presented as somatic, which the doctor assessed as being entirely somatic (SS), mental complaints, both in terms of presentation as in terms of assessment by the doctor (PP) and the cases, in which the patient expresses somatic complaints, but which the doctor thinks to be caused by factors other than the purely somatic (SP). The fourth combination, mental complaint in presentation but assessed by the doctor as purely somatic, hardly occurred; therefore we have not included it here.

The interviews in which the GHQs were taken were all carried out in a short space of time, whilst the encounters with the GPs took place over a period of 3 months. For this reason we selected for our analysis all the complaints from patients (who completed a GHQ) who in the week of the GHQ administration consulter their GP. The contacts in this week were subsequen aggregated to patient level, so that each patient only counts once $(17 \%$ of the patients in that week had either consulted their GP more than once or had presented more than one complaint during an encounter).

\section{RESULTS}

In this week 'GHQ patients' had 799 contacts. These contacts originated from 627 patients, of which 151 had one or more values missing, which led to their figures being excluded. The analysis on the week-file takes in 476 patients. The GHQ-30 was completed by all the patients involved in the research taken all together $(n=8747)$ and by the patients from the week's sample survey; the results are shown in Table 1.

Using a cut-off point between GHQ score 4 and 5, as recommended by Goldberg, ${ }^{3}, 20 \%$ of the attenders were found to be a 'case'; $12 \%$ of the random sample from the population was above this threshold. The GHQ score in the group we selected is in general higher than in the whole study: this can be explained by the fact that in our selection only the patients who consulted their GP in the same period are included.

The psychosocial problems in the group we select were characterized by the words in which the patient presented the complaint, that is to say as a 'psychosocial problem' or as a 'somatic complaint', and also by the assessment which was made by the doctor, that is to say 'purely somatic' or 'partly of a psychosocial nature'.

In a cross-tabulation the two dichotomies can be seen set against one another (Table 2).

Due to the fact that the data from the table above are 
TABLE 2 Patient's complaint (RFE) and its assessment by the GP $(n=476)$

\begin{tabular}{lccc}
\hline & Somatic RFE & Psychosocial RFE & Total \\
\hline Somatic assessment & $294(62 \%)$ & $5(1 \%)$ & $299(63 \%)$ \\
Psychosocial assessment & $125(26 \%)$ & $52(11 \%)$ & $177(37 \%)$ \\
Total & $419(88 \%)$ & $57(12 \%)$ & $476(100 \%)$ \\
\hline
\end{tabular}

based on aggregated data, this is not a record of the absolute number of encounters, but data about patients who visited their GP within the period of a week. This implies that a number of patients consulted their GPs more than once. Sixty-two per cent of all patients presented a somatic complaint which the doctor also assessed as somatic; $11 \%$ of the patients presented psychosocial complaints, which the doctor also estimated to be psychosocial. In $26 \%$ of the patients comorbidity of a psychological nature was involved, at least according to the judgement of the GP. It is worth noting that practically the same relationship between the patient's presentation and GP's assessment was found in the total file from which the week was selected.

The question which is central to this analysis is, of course, how the GHQ compares proportionately to the encounter data. The relationships between the GHQ and the patients' presentations of complaints, and between the GHQ and the assessment of the doctor, have been examined consecutively. Table 3 illustrates the situation which emerges if the patients' presentations of complaints are split into somatic on the one side and psychosocial on the other, and a cut-off point for the GHQ is established at $4 / 5$.

In the same way we can compare the GP's assessment with that of GHQ (Table 4).

Patients with psychosocial complaints have, in a majority of cases, a low GHQ score, both when based on RFE and on the assessment of the doctor. Only $39 \%(22 / 57)$ of the patients who present psychosocial problems and $28 \%(49 / 128)$ of those in whom GP assesses that psychosocial aspects played a part in their complaints, were actually GHQ cases, if we take the cut-off point to be 4 . Figure 1 shows comparable percentages obtained by increasing or decreasing the cutoff point for GHQ. Using the chosen cut-off point, the majority of the so called GHQ cases present purely somatic complaints, and the assessment of the GP in the majority of these cases is purely somatic. At this point it should be pointed out that the GHQ cases showed a higher proportion of psychosocial assessments by GPs than did the number of patients pres- enting psychosocial complaints. Most patients who do not present psychosocial problems during the consultation or whose complaints were assessed as somatic fall beneath the cut-off point for GHQ fall (Figure 2).

We shall round the analysis off by briefly considering the content of complaints.

PP-complaints* from patients with a low GHQ score (termed PP-low), differ from PP-complaints from patients with a high GHQ score (PP-high) in that the psychosocial complaint is not always expressed as the first symptom. PP-cases with a high GHQ-score always have a mental symptom as the most important one. Among the 'PP-low' cases there were half as many mental complaints mentioned as the first symptom (including requests for psychotropic drugs) as among the 'PP-high' encounters. In addition, slightly more patients with a high GHQ score appeared to present complaints which resulted in a psychiatric diagnosis; this does not mean, however, that within the 'GHQlow' group no psychiatric diagnosis at all has been established.

However, in other respects no differences were found: both 'PP-low' and 'PP-high' encounters highlighted complaints about housing and financial problems.

Which patients present an SS complaint at the consultation, but belong nevertheless to the category of 'psychiatric cases' according to the GHQ? Among the 'SS-high' contacts (in other words: somatic complaints, somatic assessment and a high GHQ score), respiratory disorders and gynecological complaints were over-represented when compared to the 'SS-low' contacts; complaints concerning the limbs and skin were rather under-represented. The complaints that one might expect, like neurovegetative complaints, and complaints related to neurology did not occur in relatively larger numbers within the SS-high group compared with SS low patients.

PP stands for mental complaint combined with a mental assessment. SS stands for a somatic complaint combined with a somatic assessment. SP stands for a somatic complaint combined with a mental assessment.

TABLE 3 Relationship between RFE (patient's complaint) and GHQ $(n=476)$

\begin{tabular}{lccc} 
& Somatic RFE & Psychosocial RFE & Total \\
\hline GHQ $\leqslant 4$ & $341(72 \%)$ & $35(7 \%)$ & $376(79 \%)$ \\
GHQ $\leqslant 4$ & $78(16 \%)$ & $22(5 \%)$ & $100(21 \%)$ \\
Total & $419(88 \%)$ & $57(12 \%)$ & $476(100 \%)$ \\
\hline
\end{tabular}


TABLE 4 Relationship between the assessment of the GP and GHQ $(n=476)$

\begin{tabular}{lccc}
\hline & Somatic assessment & Psychosocial assessment & Total \\
\hline GHQ $\leqslant 4$ & $248(52 \%)$ & $128(27 \%)$ & $376(79 \%)$ \\
GHQ $>4$ & $51(11 \%)$ & $49(10 \%)$ & $100(21 \%)$ \\
Total & $299(63 \%)$ & $177(13 \%)$ & $476(100 \%)$ \\
\hline
\end{tabular}

We mentioned the fact that some adjustments were made to the original material. This was done in order to prevent people who frequently visit their GP to be over-represented and to forestall the occurrence of differences in the intervals between GHQ administration and encounter registration. Each patient was counted once, and only encounters in the week of the interview were selected. Bearing this in mind, we can state that the same tendencies, outlined above, are to be found in the total file (considering all the encounters of all patients in a 3-month period); these measures do not improve if we take a cross-section of a day from the interview period instead of a week.

DISCUSSION

Presentation of complaints and assessments by the GP were compared with the GHQ score of the patients involved; $20 \%$ of the patients who visited their GP in a one week period were probable psychiatric cases. This figure is rather low, compared with other studies within general practice. Wilmink ${ }^{10}$ for example reports $46 \%$ GHQ cases within a random general practice population, and Von Korff et al..$^{20}$ arrived at a figure of $40 \%$

Our percentage still exceeds the number of 'cases' detected in a random population sample. The latter result is comparable to that of Finlay-Jones and Burvill, ${ }^{21}$ who reported from an Australian morbidity survey that 1 in 60 'GHQ cases' consulted their GP, compared to 1 in 130 non-cases. Goldberg et al. ${ }^{22}$ also confirmed this finding. 'Those attending a general practitioner are shown to be more psychiatrically disturbed than a random sample of the practice population.' Likewise, Berwick et al. ${ }^{23}$ report that those who score high on the GHQ are more likely to enter the medical care system.

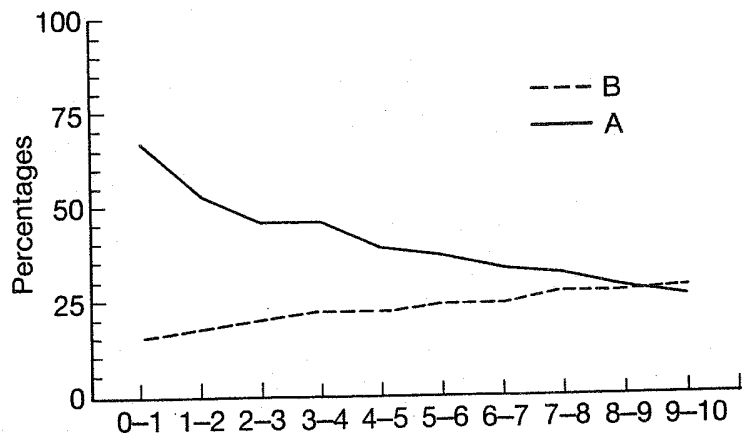

Figure 1 Percentage psychosocial RFE's who are also $G H Q$ cases $(A)$, against percentage of $G H Q$ cases who present psychosocial RFEs $(B)$, with variable cut-off points
Twelve per cent of the patients presenting during the study period presented with psychological complaints. However $37 \%$ of all complaints were considered to be not entirely somatic by the GP. Both figures are not uncommon in similar studies. Verhaak and Wennink ${ }^{24}$ report $9 \%$ psychosocial complaints (patients' own words) and $47 \%$ non-somatic assessments (by the GP) in a sample of 30 GPs who were slightly more psycho. logically minded than the sample in this study.

Wilmink ${ }^{10}$ reports that $12 \%$ patients presented with psychosocial complaints, while $26 \%$ of patients were identified as a 'mental health problem' by the GP. His positive definition of a mental health problem can be considered as somewhat stricter than our negatively formulated definition of 'not entirely somatic'.

This study was concerned with the relationship between the GHQ on the one hand and complaints/ assessments on the other. This relationship proved be rather weak in two senses: most psychosocially f .. sented complaints/psychosocial assessments were not GHQ cases, and most GHQ cases presented somatic complaints, assessed as being purely somatic. As Wilmink undertook the same kind of analyses, we may compare our results with his. Most of Wilmink's GHQ cases $(58 \%)$ were assessed by GPs as having no mental health problem. Most mental health problems (defined by the GP) were detected by the GHQ $(74 \%)$. The first result fits with the data presented in Table 4, the second does not. We must keep in mind that Wilmink found a higher proportion of GHQ cases and a lower proportion of positive assessments by the GP.

What are the consequences of these results for the evaluation of the GHQ as a screening instrument for general practice? Let us first take the GHQ as the

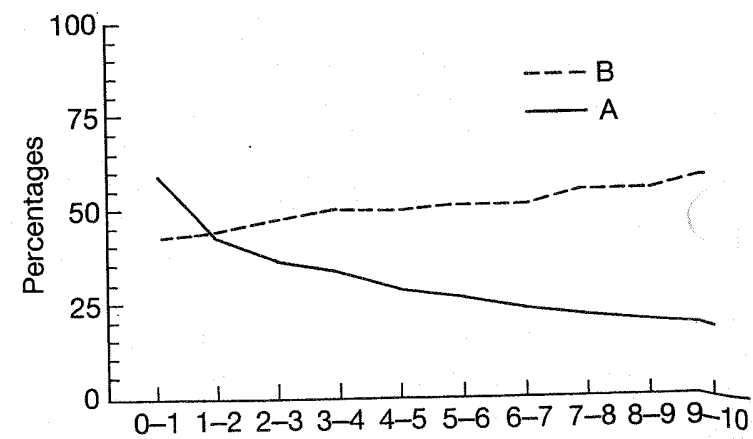

FIGURE 2 Percentage non-somatic GP assessments who are also $G H Q$ cases $(A)$ against percentage $G H Q$ cases where the $G P$ assessment is non-somatic $(B)$, with variable cut-off points 
departure point for our reflections. We are dealing here with a well-validated instrument, and we may therefore conclude that many patients who consult their GP have a psychiatric problem which they do not reveal to their GP: secondly, the GP, given a somatic presentation of complaints, sees no reason to suspect a psychiatric problem. If we look closely at the sort of complaints involved, then it becomes clear that it is difficult for the GP to find any indicators from the complaint presented by the patient. In actual fact, the somatic complaints made by patients with a high GHQ score and by those with a low GHQ score are strikingly similar. An interesting question to tackle here is the extent to which interweaving of mental and somatic problems (comorbidity) influences the proceedings. Earlier research ${ }^{24}$ has shown that in patients with comorbidity the GP tends to follow up the somatic aspects. In this respect it may be worthwhile to point to the nature of the GPs population, which is known to contain a large number of patients with chronic somatic complaints. Van der $\mathrm{Zee}^{25}$ pointed to the fact that these patients score very high on the 'VOEG', an instrument which bears some similarity to the GHQ, on which a high score indicates not psychiatric problems but chronic discomfort. In the case of the GHQ a high score might also be indicative of somatic indisposition.

Other investigations within our research group ${ }^{26}$ have indicated that it is possible to successfully teach a GP a Rogerian approach. The GPs performance proved to be restricted however to an attitude of listening and counselling; they failed to detect a possible psychiatric background to a somatic presentation. Furthermore, we should like to point out that, generally speaking, GPs tend not to make use of their patient's attendance at their surgery to carry out screening activities. Research into preventive activities carried out by GPs has shown that the average GP took the actual request made by the patient as the starting point for his treatment. ${ }^{25}$ It seems that GPs have certain reservations about the detection of diseases when there is no hint of a complaint actually in existence.

Finally, it should be pointed out that an essential condition for the execution of a screening program is the availability of a valid intervention program. Authors like Schnabel ${ }^{27}$ and Gerards ${ }^{28}$ point out that in many cases there is no reason to believe that early detection (without recognition by the patient) would prevent the problems becoming any more serious. If one accepts this viewpoint, it would seem to be undesirable to make the suspicion of psychiatric problems more explicit at an early stage. Caution is called for, because it appears there is a risk of 'mental fixation ${ }^{29}$ when problems are defined as being psychiatric when they have not been presented as such. This might be an explanation for GPs (correct) reluctance to search for problems which the patient is not apt to present spontaneously.

In summary, hidden psychiatric problems do not manifest themselves very conspicuously; a GP lacks the specialist requirements to uncover them and he does not have the intention to do so, when the patient is reluctant to present them. It is doubtful if early detection in those cases would be very sensible

If we take the GP's psychosocial caseload as a point of departure we are arrested by a striking phenomenon. The GP is in fact confronted with quite a number of patients with psychosocial problems, both explicit and non-formulated, but the majority of these patients are not classifed as psychiatric cases by the GHQ. One possible explanation for this might be the insensitivity of the GHQ for the measurement of borderline psychological problems. The fact that GPs are mostly confronted with milder psychological problems underlines the findings in the 'Nijmegen-Region Project', namely that psychosocial (indicating psychological problems of a relatively mild character, contrary to the psychological problems which belong to psychiatry) problems occur six times as often as psychiatric problems. ${ }^{30}$

How practical is the use of the GHQ as a screening device, given the evidence brought forward? In the present primary care situation, where a large group of undetected mental disorders exists (along with large groups of undetected internal, neurological, and circulatory diseases), it seems only attainable for a GP to wait for more conclusive evidence; this might be constituted by pertaining use of medical care, by accumulating evidence during next visits, etc. After all, one of the other conclusions from Wilmink's study was that the GHQ did not significantly improve the GP's prediction of cases, determined by the Present State Examination. Perhaps the combination of intuition and time (not the duration of a consultation, but the lifelong relationship with a patient in the hands of a GP), is at least as good as a screening device. Further research should be directed to a better understanding of the course of mental problems. It might perhaps in the future be possible to design screening instruments, aimed at reaching a better diagnosis when intervention might be successful, instead of the aim of detecting mental distress without looking at the possibilities of treatment.

\section{REFERENCES}

Goldberg D P, Huxley P. Mental illness in the community: the pathway to psychiatric care. London: Tavistock, 1980

${ }^{2}$ Schulberg H C, Burns B J. Mental disorders in primary care. Gen Hosp Psychiat 1988; 10: 79-87.

${ }^{3}$ Goldberg D. The detection of psychiatric illness by questionnaire. London: Oxford University Press, 1972.

${ }^{4}$ Goldberg D. Screening for disease. Lancet 1974; ii 1245-1248.

${ }^{5}$ Goldberg D. Detection and assessment of emotional disorders in a primary care setting. Int $J$ Mental Health 1979; 2: 30-48.

${ }^{6}$ Goldberg D, Bridges K. Screening for psychiatric illness in general practice: the general practitioner versus the 
screening questionnaire. J R Coll Gen Pract 1987; 1: $15-18$.

Giel R. Onderzoek naar psychische stoornissen. Een epidemiologische beschouwing. Tijdschrift Soc Gezondheids 1983;6: 198-203.

${ }^{8}$ Wright A F, Perini A F. Hidden psychiatric illness: use of the general health questionnaire in general practice. $J$ $R$ Coll Gen Pract 1987; 4: 164-167.

${ }^{9}$ Whewell P J, Gore V A, Leach C. Training general practitioners to improve their recognition of emotional disturbance in the consultation. JR Coll Gen Pract 1988; 6: 259-262.

${ }^{10}$ Wilmink F M. Patient, physician, psychiatrist. Assessment of mental health problems in primary care. Groningen: Dissertation, 1989.

${ }^{11}$ Goldberg D. Identifying psychiatric illness among general medical patients. $B$ Med $J 1985 ; 291$ : 161-162.

${ }^{12}$ Tarnopolsky A, Hand D J, Mclean E K, Roberts H, Wiggins R D. Validity and uses of a screening questionnaire (GHQ) in the community. Br J Psychiatr 1979; 134: 508-515.

${ }^{13}$ Tennant $C$. The General Health Questionnaire: a valid index of psychological impairment in Australian populations. Med J Aust 1977; 9: 392-394.

${ }^{14}$ Wilkinson M J B, Barczak P. Psychiatric screening in general practice: comparison of the general health questionnaire and the hospital anxiety depression scale. J R Coll Gen Pract 1988; 38: 311-313.

${ }^{15}$ Hodiamont P P G, Veling S H J. Een model voor het bepalen van psychiatrische prevalentie: de relatie GHQPSE. Tijdsch Psychiatr 1984; 8: 592-607.

${ }^{16}$ Broom A K, Kat B J B. Would more mental illness services help general practitioners manage their difficult patients? J R Coll Gen Pract 1981; 5: 303-307.

${ }^{17}$ Huygen F J A. A family physician's concept of mental health. In: Visser G J, Bensing J M, Gersons B P R, Maoz B, Margolis C Z. Mental Health and primary care. Dutch and Israelian experience. Utrecht: NIVEL, 1986.

${ }^{18}$ Foets M, Velden J van der, Zee J van der. Een nationale stu- die van Ziekten en verrichtingen in de huisartspraktijk. Projectvoorselt. Utrecht: NIVEL, 1986.

${ }^{19}$ Lamberts $\mathrm{H}$, Wood M (eds). ICPC, International Classification of Primary Care. Oxford: Oxford University Press, 1987.

${ }^{20}$ Von Korf M, Shapiro S, Burke J D, et al. Anxiety and depression in a primary care clinic. Arch Gen Psychiatr 1987; 44: 152-156.

${ }^{21}$ Finlay-Jones R A, Burvill P W. Contrasting demographic patterns of minor psychiatric morbidity in general practice and the community. Psychol Med 1978; 8: $455-456$.

${ }^{22}$ Goldberg D, Kay C, Thompson L. Psychiatric morbidity in general practice and the community. Psychol Med 1976; 6: 565-569.

${ }^{23}$ Berwick D M, Budman S, Damico-White J, Feldstein M, Klerman G L. Assessment of psychological morbidity in primary care: explorations with the general health in primary care: explorations with the questionnaire. J Chron

${ }^{24}$ Verhaak P F M, Wennink HJ. What complaints. Psychiat Med; In Press.

${ }^{25}$ Zee J van der, Verhaak P F M. Preventie, een kwestie van routine. Med Contact 1989; 3: 75-78.

${ }^{26}$ Bensing J M, Sluijs E M. Evaluation of an interview training course for general practitioners. Social Sci Med 1985; 20: $737-744$.

${ }^{27}$ Schnabel P. Het eigene van de geestelijke gezondheidszorg. In: Geestelijke Volksgezondheid, Ministerie van WVC, 1984.

${ }^{28}$ Gerards F M. Van kwaad tot erger? De geldigheid van de continuiteitshypothese in de psychosociale preventie. Maandblad Geestelijke Volksgezondheid 1985; 3: 243-258.

${ }^{29}$ Grol R P T M (ed). Huisarts en somatische fixatie. Utrecht: Bohn, Scheltema en Holkema, 1983.

${ }^{30}$ Furer J W, Tax B. Somatische klachten, psychiatrische symptomen en psychosociale problemen. Eindrapportage van het Regioproject geneeskunde, Katholieke Universiteit. Nijmegen: Instituut voor sociale Psychiatry, 1987. 